\title{
Silicon Plays a Key Role in Rare Earth Elements Detoxification in a Hyperaccumulator Fern
}

\author{
WEN-SHEN LIU ${ }^{1}$, YE-TAO TANG ${ }^{1,}$, , ANTONY VAN DER \\ ENT $^{2}$, RONG-LIANG QIU ${ }^{1,3}$, \\ ${ }^{1}$ School of Environmental Science and Engineering, \\ Guangdong Provincial Key Lab for Environmental \\ Pollution Control and Remediation Technology, Sun Yat- \\ sen University, Guangzhou 510006, China \\ (eestyt@mail.sysu.edu.cn) \\ ${ }^{2}$ Centre for Mined Land Rehabilitation, Sustainable Minerals \\ Institute, The University of Queensland, Australia \\ (a.vanderent@uq.edu.au) \\ ${ }^{3}$ Guangdong Laboratory for Lingnan Modern Agriculture, \\ South China Agriculture University, Guangzhou 510642, \\ China (eesqrl@mail.sysu.edu.cn)
}

The fern Dicranopteris linearis (Gleicheniaceae) is a hyperaccumulator of Rare Earth Elements (REEs), aluminium (Al) and silicon (Si). However, the physiological mechanisms to the excess of REEs and Al, and possible interactions with $\mathrm{Si}$, are currently incompletely known. In the present study, Synchrotron Radiation-based X-ray Fluorescence (XRF), Proton Induced X-ray Fluorescence Spectrometry (PIXE), Scanning Electron Microscopy-Energy Spectroscopy (SEMEDS) and a series of chemical extraction experiments were conducted to decipher the localisation and biochemistry of REEs, $\mathrm{Al}$ and $\mathrm{Si}$ in D. linearis. The results show that all the REEs (e.g., La, Ce, Pr, Nd and Y) are mainly co-localised with $\mathrm{Mn}$ and $\mathrm{Si}$ in the necrotic lesions and the epidermis of the pinnae. The extraction patterns of REEs, $\mathrm{Al}$ and $\mathrm{Si}$ in $D$. linearis pinnae indicate an existence of REEs and $\mathrm{Al}$ silicates. Furthermore, phytolith ( $\mathrm{Si}$ oxides) and phytolith $\mathrm{Al}$ are found at the epidermis of pinnae and the pericycle of veins by SEMEDS observations. Therefore, co-precipitation with $\mathrm{Si}$ is possibably responsible for the detoxification of the high concentrations of REEs and $\mathrm{Al}$ in $D$. linearis pinnae. In conclusion, we posit that Si plays a critical role in REEs and Al detoxification within the blade. The results presented here provide insights in how plant regulates high concentrations of REEs in vivo, and this knowledge is useful for developing phytotechnological applications (such as REE agromining) using this fern in REE-contaminated sites in China. 